\title{
A Description of Normal Semigroups of Endomorphisms of Proper Independence Algebras
}

\author{
João Araújo* \\ Centro de Álgebra \\ Universidade de Lisboa \\ Av. Prof. Gama Pinto 2 \\ John Fountain
Department of Matemathics \\ University of York \\ England \\ 1649-003 Lisboa, Portugal. \\ (mjoao@lmc.fc.ul.pt) \\ (jbf1@york.ac.uk)
}

January 10, 2005

\begin{abstract}
Let $\mathcal{A}$ be a strong independence algebra of finite rank with at most one constant, and let $G$ be the group of automorphisms of $\mathcal{A}$. Let $\alpha$ be a singular endomorphism of $\mathcal{A}$ and $\alpha^{G}=\langle\{\alpha\} \cup G\rangle$. We describe the elements of $\alpha^{G}$ and give additional characterisations when $\mathcal{A}$ is a proper independence algebra and $G$ is a periodic group.

Mathematics subject classification: 20M20, 20M10, 08A35.
\end{abstract}

\section{Introduction}

Let $X$ be a finite set and let $T(X)$ and $\operatorname{Sym}(X)$ be, respectively, the transformation monoid and the symmetric group on $X$. Let $\alpha \in T(X) \backslash \operatorname{Sym}(X)$

*The first author acknowledges the support of FCT and FEDER, Projecto 893 POCTI/143, and Fundação Calouste Gulbenkian. 
and consider the semigroup $S_{\alpha}$ generated by the set $\left\{\gamma^{-1} \alpha \gamma: \gamma \in \operatorname{Sym}(X)\right\}$ of all conjugates of $\alpha$. In [6] Levi and McFadden proved that

$$
S_{\alpha}=\{\beta \in T(X): \operatorname{Ker}(\gamma \alpha) \subseteq \operatorname{Ker}(\beta) \text { for some } \gamma \in \operatorname{Sym}(X)\}=\alpha^{G} .
$$

The aim of this paper is to generalise the second equality from sets to strong independence algebras of finite rank with at most one constant. In the final section, using results of the first author [2] about proper independence algebras which have a periodic group of automorphisms, we obtain the first equality and some other characterisations of $\alpha^{G}$.

In the next section we present the basic concepts concerning strong independence algebras.

\section{Preliminaries}

We assume the reader to have a basic knowledge of both the theory of independence algebras and the theory of semigroups. For independence algebras we recommend [3] and [4] as references, and for general semigroup theory we recommend [5].

The first step in the definition of independence algebras is the introduction of a notion of independence valid for universal algebras. A subset $X$ of an algebra is said to be independent if $X=\emptyset$ or if for every element $x \in X$, we have $x \notin\langle X \backslash\{x\}\rangle$; a set is dependent if it is not independent. In general, the universe of an algebra $\mathcal{A}$ is denoted by $A$. The following result is given as Exercise 6 on p.50 of [8].

Lemma 1. For an algebra $\mathcal{A}$, the following conditions are equivalent:

(1) For every subset $X$ of $A$ and all elements $u, v$ of $A$, if the element $u \in\langle X \cup\{v\}\rangle$ and $u \notin\langle X\rangle$, then $v \in\langle X \cup\{u\}\rangle$.

(2) For every subset $X$ of $A$ and every element $u \in A$, if $X$ is independent and $u \notin\langle X\rangle$, then $X \cup\{u\}$ is independent.

(3) For every subset $X$ of $A$, if $Y$ is a maximal independent subset of $X$, then $\langle X\rangle=\langle Y\rangle$.

(4) For subsets $X, Y$ of $A$ with $Y \subseteq X$, if $Y$ is independent, then there is an independent set $Z$ with $Y \subseteq Z \subseteq X$ and $\langle Z\rangle=\langle X\rangle$. 
An algebra $\mathcal{A}$ is said to have the exchange property or to satisfy [EP] if it satisfies the equivalent conditions of Lemma 1. A basis for $\mathcal{A}$ is a subset of $A$ which generates $\mathcal{A}$ and is independent. It is clear from Lemma 1 that any algebra with $[\mathrm{EP}]$ has a basis. Furthermore, for such an algebra, bases may be characterised as minimal generating sets or maximal independent sets, and all bases for $\mathcal{A}$ have the same cardinality [4, Proposition 3.3]. This cardinal is called the $\operatorname{rank}$ of $\mathcal{A}$ and is written $\operatorname{rank}(\mathcal{A})$. If $\mathcal{A}$ is an algebra satisfying $[\mathrm{EP}]$ and $\alpha$ is an endomorphism of $\mathcal{A}$, then $\operatorname{rank}(\alpha)$ is the rank of the image of $\alpha$, that is, $\operatorname{rank}(\alpha)=\operatorname{rank}(\operatorname{im}(\alpha))$.

We observe that (4) of Lemma 1 tells us that any independent subset of $A$ can be extended to a basis for $\mathcal{A}$. We also remark that if $\mathcal{A}$ satisfies [EP], then so does any subalgebra of $\mathcal{A}$.

We now define an independence algebra to be an algebra $\mathcal{A}$ which satisfies $[\mathrm{EP}]$ and also satisfies:

$[F]$ For any basis $X$ of $\mathcal{A}$ and $\alpha: X \rightarrow A$, there is an endomorphism $\bar{\alpha}$ of $\mathcal{A}$ such that $\bar{\alpha}_{\left.\right|_{X}}=\alpha$.

In any algebra $\mathcal{A}$, the set of constants is denoted by $C o n$. Let $\mathcal{A}$ be an independence algebra and let $X, Y$ be two disjoint and independent subsets of $A$. Then $\mathcal{A}$ is said to be strong if $\langle X\rangle \cap\langle Y\rangle=C$ on implies that $X \cup Y$ is an independent set. Clearly, any subalgebra of a strong independence algebra is also a strong independence algebra. Particular cases of strong independence algebras are sets and vector spaces.

We denote the endomorphism monoid of an algebra $\mathcal{A}$ by $\operatorname{End}(\mathcal{A})$. If $\eta \in \operatorname{End}(\mathcal{A})$, we say that $\eta$ is nontrivial if $\operatorname{im}(\eta) \neq C$ on. The following lemma is a slight extension of a result that follows from Section 2 of [3].

Lemma 2. Let $\mathcal{B}$ be a subalgebra of a strong independence algebra $\mathcal{A}$, let $\eta$ be a nontrivial endomorphism of $\mathcal{A}$ and $T$ be a basis for $\mathcal{B} \eta$. Then there is a basis $Y$ for $\mathcal{B}$ such that $Y \eta \backslash C$ on $=T$.

Proof. Let $Z$ be a basis for $\{b \in B: b \eta \in C o n\}$ and let $\mathcal{B}_{T}=\left\langle Z \cup\left(T \eta^{-1} \cap B\right)\right\rangle$. By Lemma $1, \mathcal{B}_{T}$ has a basis $Y=Z \cup Y_{T}$ where $Y_{T} \subseteq T \eta^{-1} \cap B$. Clearly, $Y \eta \backslash C o n=T$ and so it suffices to prove that $\mathcal{B}_{T}=\mathcal{B}$.

Clearly, it is enough to show that $B \subseteq B_{T}$ where $B$ and $B_{T}$ denote the universes of $\mathcal{B}$ and $\mathcal{B}_{T}$ respectively. Let $b \in B$; then $b \eta \in\langle T\rangle$ so that either $b \eta \in C$ on or there are elements $t_{1}, \ldots, t_{n} \in T$ such that $b \eta \in\left\langle t_{1}, \ldots, t_{n}\right\rangle$ but $b \eta \notin\left\langle t_{2}, \ldots, t_{n}\right\rangle$. In the former case, certainly $b \in B_{T}$. In the latter case, 
for each $i=1, \ldots, n$, we can write $t_{i}=b_{i} \eta$ for some $b_{i} \in B$ since $\mathcal{B} \eta=\langle T\rangle$. Hence by the exchange property,

$$
t_{1} \in\left\langle b \eta, b_{2} \eta, \ldots, b_{n} \eta\right\rangle=\left\langle b, b_{2}, \ldots, b_{n}\right\rangle \eta .
$$

Let $c \in\left\langle b, b_{2}, \ldots, b_{n}\right\rangle$ be such that $c \eta=t_{1}$ and note that $c \notin\left\langle b_{2}, \ldots, b_{n}\right\rangle$ since $t_{1}=c \eta$ is not in $\left\langle b_{2} \eta, \ldots, b_{n} \eta\right\rangle$. Hence $b \in\left\langle c, b_{2}, \ldots, b_{n}\right\rangle$ and so $b \in B_{T}$ as required.

Of course, we can take the subalgebra $\mathcal{B}$ to be the algebra $\mathcal{A}$ itself, and we obtain the following corollary which is implicit in [3].

Corollary 3. Let $\eta$ be a nontrivial endomorphism of a strong independence algebra $\mathcal{A}$. If $T$ is a basis for $\operatorname{im} \eta$, then there is a basis $Y$ for $\mathcal{A}$ such that $Y \eta \backslash C o n=T$.

The following corollary plays a key role in Section 4.

Corollary 4. Let $\mathcal{A}$ be a strong independence algebra of finite rank and let $\alpha, \beta$ be nontrivial endomorphisms of $\mathcal{A}$ with $\operatorname{Ker}(\alpha) \subseteq \operatorname{Ker}(\beta)$. Then there are automorphisms $\gamma, \delta$ of $\mathcal{A}$ and a basis $X$ for $\mathcal{A}$ such that $\left.\operatorname{Ker}(\alpha \gamma)\right|_{X} \subseteq$ $\left.\operatorname{Ker}(\beta \delta)\right|_{X}$.

Proof. By [4, Proposition 4.5(ii)], $\beta \leqslant \mathscr{R} \alpha$ and so there is an endomorphism $\eta$ with $\beta=\alpha \eta$. Let $T$ be a basis for $\operatorname{im} \beta$. Now $\operatorname{im} \beta=(\operatorname{im} \alpha) \eta$ so that by Lemma 2, there is a basis $Y$ for $\operatorname{im} \alpha$ such that $Y \eta \backslash C o n=T$. By Corollary 3, there is basis $X$ for $\mathcal{A}$ such that $X \alpha \backslash C o n=Y$. It is easy to verify that $X \beta \backslash C o n=T$.

Since $\mathcal{A}$ has finite rank, there are automorphisms $\gamma$ and $\delta$ such that $y \gamma \in X \cap y \alpha^{-1}$ for each $y \in Y$, and $t \delta \in X \cap t \beta^{-1}$ for each $t \in T$. Restricting $\alpha \gamma$ and $\beta \delta$ to $X$ we obtain transformations of $X$, and if $x_{1} \alpha \gamma=x_{2} \alpha \gamma$, then $x_{1} \alpha=x_{2} \alpha$ since $\gamma$ is an automorphism. As $\operatorname{Ker}(\alpha) \subseteq \operatorname{Ker}(\beta)$, we get $x_{1} \beta=x_{2} \beta$ and so $x_{1} \beta \delta=x_{2} \beta \delta$.

\section{The Structure of the Semigroup $\alpha^{G}$}

Throughout this section, $\mathcal{A}$ is a strong independence algebra of finite rank, and $G$ is its automorphism group. Let $\alpha \in \operatorname{End} \mathcal{A} \backslash G$. The semigroup $\langle\{\alpha\} \cup G\rangle \backslash G$ is denoted by $\alpha^{G}$. We show that $\alpha^{G}$ is regular and describe Green's relations on it. 
Proposition 5. The semigroup $\alpha^{G}$ is regular.

Proof. Let $\beta \in \alpha^{G}$. From [3, Section 3], it follows that $\operatorname{End}(\mathcal{A})$ is unit regular, that is, there is an automorphism $\gamma$ of $\mathcal{A}$ such that $\beta \gamma \beta=\beta$. Now $\gamma \beta \gamma \in \alpha^{G}$ is an inverse of $\beta$ so that $\alpha^{G}$ is regular.

Proposition 6. Let $S=\alpha^{G}$ and $\beta_{1}, \beta_{2} \in S$. Then

(1) $\beta_{1} \mathscr{R} \beta_{2}$ if and only if $\operatorname{Ker}\left(\beta_{1}\right)=\operatorname{Ker}\left(\beta_{2}\right)$;

(2) $\beta_{1} \mathscr{L} \beta_{2}$ if and only if $\operatorname{im}\left(\beta_{1}\right)=\operatorname{im}\left(b_{2}\right)$;

(3) $\beta_{1} \mathscr{D} \beta_{2}$ if and only if $\operatorname{rank}\left(\beta_{1}\right)=\operatorname{rank}\left(\beta_{2}\right)$;

(4) $\mathscr{D}=\mathscr{J}$.

Proof. Parts (1) and (2) follow from [5, Proposition II.4.5] and [4, Corollary 4.6].

We now prove $(3)$. Two $\mathscr{D}$-related elements in $S$ are also $\mathscr{D}$-related in $\operatorname{End}(\mathcal{A})$ and so have the same rank.

On the other hand, since $S$ is regular, if $\beta_{1}, \beta_{2} \in S$, then there are idempotents $\varepsilon_{1}, \varepsilon_{2}$ in $S$ with $\beta_{i} \mathscr{L} \varepsilon_{i}$ for $i=1,2$. Hence if $\beta_{1}, \beta_{2}$ have the same rank, then so do $\varepsilon_{1}, \varepsilon_{2}$ and it suffices to prove that $\varepsilon_{1} \mathscr{D} \varepsilon_{2}$. Since $\operatorname{rank}\left(\varepsilon_{1}\right)=$ $\operatorname{rank}\left(\varepsilon_{2}\right)$ and $\mathcal{A}$ has finite rank, there is an automorphism $\gamma$ of $\mathcal{A}$ which maps $\operatorname{im}\left(\varepsilon_{1}\right)$ isomorphically onto $\operatorname{im}\left(\varepsilon_{2}\right)$. Now $\varepsilon_{1} \gamma \in S, \operatorname{Ker}\left(\varepsilon_{1} \gamma\right)=\operatorname{Ker}\left(\varepsilon_{1}\right)$ and $\operatorname{im}\left(\varepsilon_{1} \gamma\right)=\operatorname{im}\left(\varepsilon_{2}\right)$ so that by (1) and (2), $\varepsilon_{1} \mathscr{R} \varepsilon_{1} \gamma \mathscr{L} \varepsilon_{2}$. Thus $\varepsilon_{1} \mathscr{D} \varepsilon_{2}$ as required.

Finally, if $\beta_{1} \mathscr{J} \beta_{2}$, then $\beta_{1}, \beta_{2}$ are also $\mathscr{J}$-related in $\operatorname{End}(\mathcal{A})$ so that by $[4$, Proposition 4.5(iii) and (v)], they have the same rank, and hence by (3), they are $\mathscr{D}$-related in $S$. Part (4) follows.

The next corollary follows immediately from this lemma.

Corollary 7. Every ideal of $\alpha^{G}$ is a principal ideal and the principal ideals are the sets

$$
I_{r}=\left\{\beta \in \alpha^{G}: \operatorname{rank}(\beta) \leqslant r\right\},
$$

for $r=0,1, \ldots, \operatorname{rank}(\mathcal{A})-1$. 


\section{A description of the elements of $\alpha^{G}$}

From now on, $\mathcal{A}$ always denotes a strong independence algebra of finite rank with at most one constant. Thus, in the algebras under consideration, $C o n=$ $\emptyset$ or $C$ on $=\{0\}$.

In this section, we describe the semigroup $\alpha^{G}$ by specifying its elements. For the trivial endomorphism $\zeta$ which maps every element to zero, it is clear that $\zeta^{G}=\{\zeta\}$, so we may assume that $\alpha$ is nontrivial. Next we determine under what conditions $\zeta$ is a member of $\alpha^{G}$.

Lemma 8. Let $\mathcal{A}$ be a strong independence algebra of finite rank $n$ with $C$ on $=\{0\}$, and let $\beta$ be a nontrivial endomorphism of $\mathcal{A}$ such that $\{0\} \neq 0 \beta^{-1} \varsubsetneqq \mathcal{A}$. Then there is an automorphism $\gamma$ of $\mathcal{A}$ such that $0 \beta^{-1} \varsubsetneqq$ $0(\beta \gamma \beta)^{-1}$.

Proof. Clearly, $0 \beta^{-1} \subseteq 0(\beta \gamma \beta)^{-1}$.

If $0 \beta^{-1} \cap \operatorname{im} \beta \neq\{0\}$, let $y=x \beta$ be a non-zero element of $0 \beta^{-1} \cap \operatorname{im} \beta$. Then $x \notin 0 \beta^{-1}$, so $0 \beta^{-1} \varsubsetneqq\left\langle\{x\} \cup 0 \beta^{-1}\right\rangle \subseteq 0\left(\beta^{2}\right)^{-1}$.

If $0 \beta^{-1} \cap \operatorname{im} \beta=\{0\}$, let $y$ and $z$ be non-zero elements of $\operatorname{im} \beta$ and $0 \beta^{-1}$ respectively. Then $\{y, z\}$ is independent, and so there is an automorphism $\gamma$ of $\mathcal{A}$ with $y \gamma=z$. Let $y=x \beta$. Then $x \in 0(\beta \gamma \beta)^{-1} \backslash 0 \beta^{-1}$ so that $0 \beta^{-1} \varsubsetneqq 0(\beta \gamma \beta)^{-1}$ as required.

Proposition 9. Let $\mathcal{A}$ be a strong independence algebra of finite rank with Con $=\{0\}$. If $\alpha$ is a nontrivial endomorphism of $\mathcal{A}$, then

$$
\zeta \in \alpha^{G} \text { if and only if } 0 \alpha^{-1} \neq\{0\} .
$$

Proof. If $\zeta \in \alpha^{G}$, then $\zeta=\gamma_{1} \alpha \gamma_{2} \ldots \alpha \gamma_{n}$ for some automorphisms $\gamma_{1}, \ldots, \gamma_{n}$. Clearly, $\gamma_{n}$ can be taken to be the identity, and we can assume that there is an element $x$ in $\mathcal{A}$ such that $x \gamma_{1} \alpha \gamma_{2} \ldots \alpha \gamma_{n-1} \neq 0$. Thus $0 \alpha^{-1} \neq\{0\}$.

If $0 \alpha^{-1} \neq\{0\}$, then by repeated applications of Lemma 8 we obtain $\zeta \in \alpha^{G}$.

We now consider the nontrivial elements of $\alpha^{G}$. We introduce the following notation:

$$
\alpha^{G}(\zeta)=\left\{\begin{array}{l}
\alpha^{G} \text { if } \text { Con }=\emptyset \\
\alpha^{G}(\zeta)=\alpha^{G} \cup\{\zeta\} \text { if Con }=\{0\} .
\end{array}\right.
$$

Our result is analogous to, and generalises, the description of $\alpha^{G}$ given in [6] for the case when $\alpha$ is simply a mapping of a finite set to itself. 
Theorem 10. Let $\mathcal{A}$ be a strong independence algebra of finite rank with at most one constant. Let $\alpha \in \operatorname{End}(\mathcal{A}) \backslash A u t(\mathcal{A})$ be a non-trivial endomorphism. Then

$$
\alpha^{G}(\zeta)=\{\beta \in \operatorname{End}(\mathcal{A}): \operatorname{Ker}(\gamma \alpha) \subseteq \operatorname{Ker}(\beta) \text { for some } \gamma \in G\} .
$$

Proof. If $C o n=\{0\}$, then clearly, $\zeta$ is in both sets.

Suppose that $\beta$ is a nontrivial endomorphism such that $\operatorname{Ker}(\gamma \alpha) \subseteq \operatorname{Ker}(\beta)$ for some $\gamma \in G$. By Corollary 4, there are automorphisms $\theta, \delta$ of $\mathcal{A}$ and a basis $X$ for $\mathcal{A}$ such that $\left.(\gamma \alpha \theta)\right|_{X},\left.(\beta \delta)\right|_{X} \in T(X)$ and $\operatorname{Ker}\left(\left.(\gamma \alpha \theta)\right|_{X}\right) \subseteq \operatorname{Ker}\left(\left.(\beta \delta)\right|_{X}\right)$. Hence by $[6$, Proposition 4], we have

$$
\left.(\beta \delta)\right|_{X} \in\left\langle\left.(\gamma \alpha \theta)\right|_{X}, \operatorname{Sym}(X)\right\rangle \backslash \operatorname{Sym}(X) .
$$

It follows easily that

$$
\beta=(\beta \delta) \delta^{-1} \in(\gamma \alpha \theta)^{G}=\alpha^{G}
$$

Now suppose that $\beta \in \alpha^{G}$ and $\beta \neq \zeta$. Then $\beta=\gamma \alpha \gamma_{1} \alpha \ldots \gamma_{n-1} \alpha \gamma_{n}$ for some $n$ and some $\gamma, \gamma_{1}, \ldots, \gamma_{n} \in G$. Clearly, $\operatorname{Ker}(\gamma \alpha) \subseteq \operatorname{Ker}(\beta)$ and the proof is complete.

As an easy consequence we have the following.

Corollary 11. Let $\mathcal{A}$ be a strong independence algebra of finite rank with at most one constant. Let $\alpha \in \operatorname{End}(\mathcal{A}) \backslash A u t(\mathcal{A})$ be a non-trivial endomorphism. Then

$$
\alpha^{G}(\zeta)=\left\{\beta \in \operatorname{End}(\mathcal{A}): \operatorname{Ker}(\beta)=\operatorname{Ker}(\varepsilon), \varepsilon \in E\left(\alpha^{G}\right)\right\} .
$$

Proof. Let $\beta \in \alpha^{G}$. As $\alpha^{G}$ is regular, it follows that $\beta$ is $\mathscr{R}$-related to some idempotent, $\varepsilon \in \alpha^{G}$, and hence $\operatorname{Ker}(\beta)=\operatorname{Ker}(\varepsilon)$.

Conversely, if $\operatorname{Ker}(\beta)=\operatorname{Ker}(\varepsilon)$ for some idempotent $\varepsilon \in \alpha^{G}$, then by Theorem 10, we have $\beta \in \varepsilon^{G} \subseteq \alpha^{G}$.

\section{$5 \quad \operatorname{Aut}(\mathcal{A})$-Normal Semigroups}

Let $S$ be a monoid with group of units $G$. We say that $T \leqslant S$ is $G$-normal if $g^{-1} T g \subseteq Y$ for all $g \in G$. 
To conclude the paper, we consider analogues of the other characterisations of $\alpha^{G}$ given in [6] when $\alpha \in T(X) \backslash \operatorname{Sym}(X)$ and $X$ is a finite set. Let $E(S)$ denote the set of idempotents of a semigroup $S$. The results obtained in [6] can be described as follows: let $X$ be a set, let $\alpha \in T(X) \backslash \operatorname{Sym}(X)$ and let $S_{\alpha}=\left\langle\gamma^{-1} \alpha \gamma: \gamma \in \operatorname{Sym}(X)\right\rangle$; then

$$
\begin{aligned}
S_{\alpha} & =\left\langle E\left(S_{\alpha}\right)\right\rangle \\
& =\left\langle E\left(\alpha^{G}\right)\right\rangle \\
& =\alpha^{G} \\
& =\{\beta \in T(X): \operatorname{Ker}(\gamma \alpha) \subseteq \operatorname{Ker}(\beta) \text { for some } \gamma \in \operatorname{Sym}(X)\}
\end{aligned}
$$

Naturally, we may ask if a corresponding result holds for a strong independence algebra $\mathcal{A}$ of finite rank with at most one constant. For such a result we would replace $\operatorname{Sym}(X)$ by $G=\operatorname{Aut}(\mathcal{A}), T(X)$ by $\operatorname{End}(\mathcal{A})$, and adopting and extending the convention of the previous section, $\alpha^{G}$ by $\alpha^{G}(\zeta)$ and $S_{\alpha}$ by $S_{\alpha}(\zeta)$.

For any strong independence algebra $\mathcal{A}$ of finite rank with at most one constant we have the result corresponding to the last equality by Theorem 10 . However, for the other equalities, we are able to get the corresponding results only under further restrictions on $\mathcal{A}$. To describe these restrictions we start by defining an endomorphism $\alpha$ of $\mathcal{A}$ to be proper if there is a basis $X$ for $\mathcal{A}$ such that $X \alpha$ is a basis for $\operatorname{im}(\alpha)$. We say that a strong independence algebra with at most one constant is proper if all its endomorphisms of rank at least 1 are proper.

We remark that strong independence algebras without constants and vector spaces over fields are examples of proper independence algebras (see [2]).

In $[2$, Theorem 3.1], it is proved that if $\mathcal{A}$ is a proper independence algebra of finite rank, then $\alpha^{G}=\left\langle E\left(\alpha^{G}\right)\right\rangle$. Using a technique devised by McAlister [8], it is also shown in [2, Theorem 4.1] that for such an algebra $\mathcal{A}$, if $G$ is a periodic group, then

$$
\left\langle E\left(S_{\alpha}\right)\right\rangle=\left\langle E\left(\alpha^{G}\right)\right\rangle .
$$

Hence, when $G$ is a periodic group, we have

$$
S_{\alpha} \subseteq \alpha^{G}=\left\langle E\left(\alpha^{G}\right)\right\rangle=\left\langle E\left(S_{\alpha}\right)\right\rangle \subseteq S_{\alpha}
$$

Putting these results together with Theorem 10 we thus obtain the following theorem. 
Theorem 12. Let $\mathcal{A}$ be a proper independence algebra of finite rank such that $G=\operatorname{Aut}(\mathcal{A})$ is a periodic group. Let $\alpha \in \operatorname{End}(\mathcal{A}) \backslash G$ be nontrivial. Then

$$
\begin{aligned}
S_{\alpha}(\zeta) & =\left\langle E\left(S_{\alpha}(\zeta)\right)\right\rangle \\
& =\left\langle E\left(\alpha^{G}(\zeta)\right)\right\rangle \\
& =\alpha^{G}(\zeta) \\
& =\{\beta \in \operatorname{End}(\mathcal{A}): \operatorname{Ker}(\gamma \alpha) \subseteq \operatorname{Ker}(\beta) \text { for some } \gamma \in G\}
\end{aligned}
$$

We observe that, in view of the complexity of the main proof in [1], the problem of describing $S_{\alpha}$ when $G$ is not periodic seems to be rather difficult.

\section{References}

[1] J. Araújo, F. C. Silva, Semigroups of linear endomorphisms closed under conjugation, Comm. Algebra 28 (2000), 3679-3689.

[2] J. Araújo, Normal semigroups of endomorphisms of proper independence algebras are idempotent generated, Proc. Edinburgh Math. Soc. 45 (2002), 205-217.

[3] J. Fountain, A. Lewin, Products of idempotent endomorphisms of an independence algebra of infinite rank, Math. Proc. Camb. Phil. Soc. 114 (1993), 303-319.

[4] V. Gould, Independence algebras, Algebra Universalis 33 (1995), 294318.

[5] J. M. Howie, An Introduction to Semigroup Theory, Academic Press, London, (1976).

[6] I. Levi and R. McFadden, $S_{n}$-normal semigroups Proc. Edinburgh Math. Soc. 37 (1994), 471-476.

[7] D. B. McAlister, Semigroups generated by a group and an idempotent, Comm. Algebra 26 (1998), 515-547.

[8] R.N. McKenzie, G. F. McNulty and W. F. Taylor, Algebra, lattices, varieties, Vol. I Wadsworth, Monterey, (1983). 